\title{
EL TERRITORIO COMO BASE DE UNA VIOLENCIA ESPACIAL
}

\author{
THE TERRITORY AS A BASE OF A SPACE VIOLENCE
}

\author{
Susana García Bujalance \\ Escuela de Arquitectura de Málaga (España)
}

Recibido: 20-05-2012

Aceptado: 26-06-2012

Resumen: El territorio se ha convertido en la base del desarrollo de la civilización moderna. El consumo de sus recursos naturales, culturales y sociales está en el origen de la contradicción de los valores ilustrados de la sociedad tardomoderna, que hablando el lenguaje de la libertad, la igualdad y la solidaridad, genera una tensión en la mujer y el hombre común, de carácter espiritual. La guerra está dejando de ser percibida como el enfrentamiento entre naciones, para experimentarse como la desincronización entre el individuo y su entorno. La violencia espacial surge como una nueva forma de violencia asociada a la transformación del territorio, como principio del desarrollo moderno.

Palabras-clave: territorio, violencia espacial, sociedad, urbanismo, desarrollo, modernidad.

\begin{abstract}
The territory has become the basis for development of modern civilization. The consumption of natural, cultural and social resources is at the origin of the contradiction of late modern society illustrated values, speaking the language of freedom, equality and solidarity, creates a tension in the women and the common man, of a spiritual nature. The war is ceasing to be perceived as the confrontation between nations to be experienced as the desynchronization between the individual and the environment. Spatial violence emerges as a new form of violence associated with the transformation of the territory, as a principle of modern development.
\end{abstract}

Key-words: land, space violence, society, urban planning, development, modernity. 


\section{Punto de partida. Enunciado de un nuevo marco conceptual.}

Hoy en el mundo civilizado, vivimos una guerra sorda, una hostilidad latente entre grupos opuestos. En el contexto de crisis en que se desarrolla esta parte de la historia reciente desde la caída del muro de Berlín, la guerra ha modificado su sentido.

La violencia física entre naciones que en la primera mitad del siglo XX se conocía por guerra, ha pasado a denominarse conflicto armado, acción preventiva $u$ ofensiva ante un ataque inminente.

Este hecho alerta -más que sobre el poder de un lenguaje que pretende ocultar la contradicción moral de una sociedad pretendidamente democrática y libre que no quiere reconocer la violencia que aplica para lograr dichos objetivos-, sobre el desplazamiento que de una manera más bien oculta se está produciendo en los objetivos y el campo de batalla donde se produce la guerra.

Guerra, según la Real Academia Española de la Lengua, es la desavenencia y rompimiento de la paz entre dos o más potencias o la lucha armada entre dos o más naciones o entre bandos de una misma nación.

Pero también es, en su cuarta acepción, la lucha o combate, aunque sea en sentido moral. Y en su quinta acepción la oposición de una cosa con otra.

En una guerra, la oposición entre las partes genera violencia, que significa aplicar medios para a través de la fuerza -no necesariamente armada-, vencer la resistencia de una parte contra la otra.

Podríamos convenir que hoy se está produciendo una guerra entre el hombre y la mujer contemporáneos, y un conjunto de elementos heterogéneos en su naturaleza que generan una violencia que trata de someterlos a sus abstractos intereses y a una lógica ajena a su condición humana. Pero, ¿dónde se desarrolla esa guerra?

Es posible que estemos asistiendo a la consolidación de un campo de batalla multiescalar en el que el territorio es el escenario de una violencia espacial que genera una profunda desincronización entre la naturaleza humana y su entorno.

\section{Génesis y origen de la violencia espacial.}

Si acudimos de nuevo a la Real Academia Española de la Lengua, encontramos que territorio, en tanto que terreno, es el campo o esfera de acción en que con mayor eficacia puede mostrarse la índole o las cualidades de personas o cosas.

Y es que a nuestro entender, el territorio no es un espacio geográfico sobre el que se asientan las estructuras humanas. El territorio es una construcción colectiva, un diálogo del ser humano con la naturaleza a lo largo de la historia.

THÉMATA. Revista de Filosofía, $\mathrm{N}^{\circ} 48$ julio-diciembre (2013) pp.: 37-48 doi: 10.12795/themata.2013.i48.03 
Si buscamos la razón de los asentamientos humanos a lo largo de su evolución, siempre encontraremos una regla o una norma que vincula esta decisión a la eficacia de su ubicación, o a la conexión simbólica que una determinada comunidad podía manifestar respecto de un determinado lugar.

Durante miles de años, las primeras formaciones clánicas, fueron reconociendo el terreno que habitaban en un proceso de antropización del mismo.

En los estudios de Gianfranco Caniggia (Caniggia, 1976) (Caniggia \& Maffei, 1979) se expone un modelo tipológico que explicaría los asentamientos urbanos a lo largo de la historia. Pero al mismo tiempo, sugiere la existencia de un tipo que tendría que ver con el concepto de territorio. Según este modelo, el ser humano establecería un primer contacto con el entorno, un registro relacionado con la necesidad de saber y saberse en el mundo. La humanidad, en su discurrir a lo largo de los siglos, ha necesitado apropiarse del territorio, conocerlo y dominarlo. Aquel primer despliegue en busca de alimento y materiales líticos, supuso de algún modo, un modelo de arraigo y pertenencia.

A lo largo de milenios el ser humano recorrió el territorio registrándolo, habitándolo y haciéndolo suyo. Y es que, según G. Zarone "habitar quiere decir tener raices en un lugar, y por ello tener asiento en un sitio, y esto a su vez (quiere decir) existir, superarse manteniéndose ligado a aquel fundo en que se ha tomado plaza para siempre”. (Zarone, 1993, p. 11)

Antes de habitar la casa, las comunidades habitaron el territorio y lo estructuró en una forma, en un tipo. Caniggia (Caniggia \& Maffei, 1979) enuncia la siguiente definición de ese tipo:

"El tipo territorial es el concepto de territorio que cada hombre, perteneciente a una época y a un lugar, asume: la conciencia espontánea del área en que vive y que engloba unitariamente un modo de recorrer el territorio, de elegir un lugar donde asentarse, de implantar su propia actividad productiva y, finalmente, de comprender un posterior lugar dotado de la modalidad suficiente para ser sede de cambio, de relación, de encuentro con otros hombres de otra entidad territorial. Es sobre todo, un concepto comprensivo de una entidad dimensional, de una cantidad de territorio: aquella que, de época en época y de lugar en lugar, el hombre acepta como dimensión en que ejercita la propia vida, y a la cual tiene conciencia de pertenecer"

En cierto modo, este sentido de pertenencia de los seres humanos al territorio que habitaban, no estaba exento de un proyecto vital que vinculaba al clan.

Así, durante siglos, los habitantes de cada determinado territorio concentraron sus fuerzas en la construcción colectiva de una sociedad, de una economía, de una cultura y también, de una identidad. El conocimiento, el desarrollo de nuevas tecnologías y habilidades, hizo que la sociedad se empeñase en el control de todos los inconvenientes y obstáculos que se oponían a su dominio, y esto también afectó a su relación con las infraestructuras ambientales que lo definían, como los ríos, las sierras, los valles o las costas. Estos elementos 
dejaron de ser la razón y ser de las ciudades que se habían desarrollado en un determinado lugar, para convertirse en base para la explotación de los recursos necesarios para su sostenimiento.

En este sentido, el vaciado por agotamiento concepto de la sostenibilidad, no tiene cabida en su aplicación a la ciudad. La ciudad no puede ser sostenible, pues para su mantenimiento es necesaria la aplicación de una energía que consume los recursos no renovables de la naturaleza.

La naturaleza, como sistema, es sostenible en tanto que la energía necesaria para su mantenimiento es la energía inagotable del sol. La ciudad como sistema, no es sostenible pues, hasta que la humanidad sea capaz de generar una energía inagotable y que no genere residuos, ésta necesita de la aplicación de un trabajo que obtiene su energía de las fuentes naturales agotables.

En tanto que el hombre estaba más cerca de su comportamiento como animal, la integración de sus modos de vida en el sistema natural era posible. Cuando su capacidad social ha primado sobre su condición animal, ha requerido de una fuerza de cohesión que se ha materializado en ciudades cada vez más extensas y complejas que requieren cantidades cada vez más desmesuradas de energía para su mantenimiento.

Así, la sostenibilidad del sistema ciudad, sólo es posible a costa del agotamiento del sistema naturaleza. He ahí la base de la contradicción de una sociedad que se reconoce en los valores de paz urbana definidos en la Ilustración y consolidados durante la modernidad, pero que niega la agresión continuada a la naturaleza sobre la que se asienta.

Podemos ubicar el inicio de la aceleración de este proceso en el desarrollo industrial del siglo XVIII. Con el progreso de la tecnología con base en los avances científicos de la Ilustración, se inició una expropiación de los recursos naturales que durante milenios habían sido la base de la cultura y la subsistencia de muchos pueblos. Y este expolio fue posible por el desarrollo de un marco normativo que favoreció la prevalencia de los valores de propiedad frente a los valores del uso colectivo.

En el año 1725, dos ingenieros alemanes con conocimientos de la tecnología necesaria para fabricar hoja de lata, pidieron autorización a la Real Junta de Gobierno para poder fabricarla en el malagueño valle del Genal. La razón, para tan por otra parte tortuosa ubicación, era la posibilidad de explotación sistemática de los recursos tradicionales de aquel territorio: el agua del río Genal, el mineral del yacimiento de magnetita y el carbón obtenido de la tala de los centenarios robles y castaños de la Sierra Bermeja y de las Nieves.

En 1726 Felipe V firmó un Real Privilegio que les concedía la exclusiva para la fabricación de hoja de lata durante los siguientes quince años. Así nació la Real Fábrica de Hoja de Lata de San Miguel, la cual fue posible gracias a los privilegios de carácter arancelario y comercial que aquella concesión real implicaba: libertad para la tala de árboles, posibilidad de requisar locales y vi-

THÉMATA. Revista de Filosofía, $\mathrm{N}^{\circ} 48$ julio-diciembre (2013) pp.: 37-48

doi: 10.12795/themata.2013.i48.03 
viendas, primacía para el aprovechamiento de las aguas del río con el objetivo de accionar las máquinas de la Real Fábrica, y el uso de Armas Reales (Gómez Zotano, 2003).

Así, el primer intento de industrialización del territorio malagueño no fue en la ciudad de Málaga, donde la demanda de hojalata podía haber sido mayor y donde se disponía de un puerto para su transporte y comercialización, sino en el pequeño pueblo de Júzcar, el cual por su ubicación estratégica permitía un mayor aprovechamiento de los recursos. No obstante, fue la ciudad de Málaga la que se benefició durante el corto periodo de tiempo que duró aquella explotación, de los beneficios de aquella industria, pues fue a través de su estructura urbana, comercial y administrativa, que se introdujo aquella producción en el mercado global.

En este caso concreto, poco habitual por otra parte, la explotación no duró mucho, pues las condiciones del privilegio fueron tan desastrosas para la sensible población que vivía en el valle, que la falta de agua para los cultivos tradicionales de la población autóctona durante los periodos estivales a causa del uso por parte de la fábrica, generaron un potente rechazo de la población, que devino en constantes saboteos a la fábrica, que finalmente cerró a los pocos años de su apertura.

Más allá del fracaso, este ejemplo ilustra el modo en que a partir de la Ilustración, el desarrollo comercial y tecnológico nacido de un contexto que favorecía la libertad del hombre frente a los privilegios de la monarquía y la nobleza, y el conocimiento de las leyes de la naturaleza frente al poder inescrutable de Dios, no fue espontáneo sino que requirió de la intervención de un poder que mediante la elaboración de normativas y reglamentos, propició este cambio.

Así, el poder aceleró y alteró un cambio estructural en la relación de las comunidades con su territorio. Hasta entonces, este había sido la base del desarrollo de una cultura, y de un modo de subsistencia que a pesar de que a lo largo de los siglos había ido consumiendo cada vez más recursos naturales, había permanecido en un cierto equilibrio. Aunque existían títulos de propiedad -sobre todo a partir de la concesión de tierras que con los Repartimientos los Reyes Católicos otorgaron a las clases que habían apoyado la Reconquista- lo que caracterizaba estos modos de vida de carácter más rural y en equilibrio con el medio era el uso comunal de las tierras y los recursos naturales, que eran considerados colectivos.

Con la variación de este sistema a través de la concesión de los recursos tradicionales que vinculaban a la población al territorio, a una burguesía comercial e industrial, se rompía el lenguaje dominante de lo común para introducir el dominio de la posición por encima de los valores tradicionales del uso y el aprovechamiento del suelo. 
Lo que aconteció después no es más que la crónica de un proceso que no dejó de incrementar esta tendencia.

El resultado de todo ello es el hecho de que el territorio, antaño base de la cultura, la identidad y la subsistencia de los pueblos a lo largo de la historia, se convirtió en la base para la explotación sistemática de los recursos agotables con el objeto de permitir y favorecer el desarrollo de una sociedad cada vez más sofisticada en la definición de sus libertades y exigencias de consumo.

Con la incorporación de nuevas tecnologías, el aprovechamiento de los recursos es cada vez más amplio. Así, en estos momentos podemos hablar del paisaje como recurso atractivo para el consumo de las masas que exigen cada vez dosis más altas de experiencias que les compensen el estrés al que les somete una ciudad que ya no es ciudad sino que se ha desparramado por el territorio.

El territorio ha dejado de ser percibido por el hombre y la mujer como la base de su hábitat, para ser objeto de valor y plus valor. Así, la localización estratégica del suelo a los nodos principales de las infraestructuras de conexión y dotación, los convierte en objeto del deseo de una nueva forma de especulación desgeoreferenciada global, que hoy al igual que que en el ejemplo de la Real Fábrica de Hoja de Lata de San Miguel, encuentra en la elaboración de normativas y reglamentos específicos, el marco legislativo que le permite a los agentes financieros globales el aprovechamiento de estos recursos territoriales, culturales e históricos.

El problema que se nos presenta es que la población no suele ser consciente del expolio que se está produciendo a un valor agotable como es el de su territorio propio. La experiencia virtual proporcionada por la velocidad de los medios de transporte y por las tecnologías de la comunicación en red, así como la aceptación del lenguaje de la propiedad como único posible, hace que desaparezca ante nuestros propios ojos la condición de constructo histórico, cultural y social del territorio. No podemos nombrar lo que no vemos. Y no vemos lo que ha desaparecido del imaginario colectivo.

El proceso de industrialización, propició aglomeraciones de suburbios en las grandes ciudades occidentales que a lo largo del siglo XIX y XX tuvieron que acoger, sin la infraestructura necesaria, a miles de trabajadoras que se concentraban en torno a las fábricas. Ésta situación generó efectos de carácter principalmente material como insalubridad, hacinamiento o falta de dotaciones. Hoy, estos problemas se han resuelto en gran parte, gracias al desarrollo de una técnica de la planificación territorial que, como en el caso de España, dotó de las principales infraestructuras y equipamientos necesarios a las ciudades a partir de los años 80, con una generación de planes urbanísticos cuyo objetivo fue resolver los problemas producidos por un desarrollo urbano voraz y no planificado. Así pues, podríamos convenir que los efectos que la postindus- 
trialización genera a los usuarios de los entornos metropolitanos es de carácter espiritual.

Esta secuela no es más que el reflejo de la guerra que cada individuo experimenta al intentar sincronizar los tiempos de su vida con los tiempos exigidos por una ciudad que en constante agresión al medio natural que la soporta, ha explotado transformándose en territorio.

Como consecuencia de este consumo del territorio como base del desarrollo progresivo de la modernidad, podemos identificar en el proceso urbano de la segunda mitad del siglo XX y la primera parte del siglo XXI, podemos reconocer tres tendencias importantes.

Por un lado, se ha producido en las últimas décadas una polarización de espacios atractores, frente a áreas que se han convertido en lugares de abastecimiento y vertido. Mientras existen áreas metropolitanas que aglutinan todos los servicios y representatividad, encontramos áreas que han quedado como zonas de abastecimiento de recursos humanos, sociales y naturales, pero también como espacios de vertido material, energético y humano de las zonas más activas del territorio. Lugares como grandes vertederos, centrales nucleares o cárceles, son habitualmente ubicadas en lugares interiores desplazados de los circuitos atractores.

Por otra parte se ha producido en todos los grandes núcleos urbanos, una conurbación difusa que se expande por el territorio ocupando grandes áreas antes agrícolamente productivas. Este modelo de ocupación está caracterizado por el despliegue de múltiples piezas residenciales o de usos productivos urbanos -suelos industriales, polígonos de almacenamiento de productos, industria escaparate, parques tecnológicos, parques empresariales, etc-, que se ubican de modo inconexo entre sí y con la ciudad central. Para su funcionamiento se requieren grandes infraestructuras de transporte y también de abastecimiento hídrico, sanitario, energético y de servicios.

Según Bernardo Secchi (Secchi, 1986), durante la primera parte del siglo XX la ciudad vivió un fenómeno de ampliación y crecimiento de los centros urbanos hacia el territorio que garantizó una centralidad difusa de éstos hacia las zonas periféricas. A través de esta centralidad se ejerció el control sobre el territorio desde las necesidades urbanas. En oposición a esto, durante la segunda parte de ese siglo se ha pasado a una urbanidad difusa en la que la deslocalización y la ocupación puntual de la extensión territorial ha terminado por dibujar una continuidad sin centro.

A estas dos tendencias formales, sumamos una tercera de carácter interpretativo. Entendemos que las ciudades y sus conurbaciones, se han convertido en objetos de mercado que atienden a las mismas reglas de competitividad y publicidad que los productos de consumo. La "marca" de las ciudades, señala la maniobra competitiva que ha convertido a los territorios en objetos de consumo.

THÉMATA. Revista de Filosofía, No 48 julio-diciembre (2013) pp.: 37-48 doi: 10.12795/themata.2013.i48.03 
Así, el sistema de la economía globalizada, se ha apropiado de los elementos que han sido construidos socialmente a lo largo de la historia, en una explotación que aspira al máximo lucro con la menor inversión posible. Se han aprovechado las condiciones de localización y las conexiones o la urbanización previa mientras se agotan los recursos mejores sin contemplaciones hacia el lugar. Esta práctica evidencia sus efectos en los territorios donde antes se produce la escasez de los recursos, como por ejemplo los litorales marítimos o los espacios de valor natural o paisajístico. Éstos suponen los territorios más deseables por su capacidad de generar plusvalías globales, convirtiéndose como las monedas, en objeto de uso. El territorio, esa nueva condición de urbanidad, ya no es lo que supone como constructo histórico, sino que se ha convertido en portador de valor.

Desde que la ordenación del territorio se ha incorporado a la práctica política como herramienta técnica al servicio de los intereses geoeconómicos, las ciudades se han transformado en simples contenedores de productos de consumo. El urbanismo ha sido el instrumento disciplinar al servicio de esta democratización de la banalidad, que nunca ha mostrado interés por lo auténtico y específico de cada sociedad y cada territorio, pues ha expulsado lo que le confiere una vida auténtica: los habitantes y su relación cultural y social con él.

Y es que lo que está en juego, precisamente, es la habitabilidad. Esa cualidad que ha perdido énfasis en el camino de la conversión de los primeros asentamientos de las hordas del paleolítico en macrociudades expandidas por el territorio, en el que éste ha dejado de ser base para la subsistencia y soporte de la cultura y los asentamientos, para convertirse en el papel en blanco sobre el que se dibuja una nueva estrategia de economía global que apoyada en el principio de posesión, se basa hoy en el consumo de bienes y experiencias, y la imagen.

\section{El objeto de la violencia.}

Tras esta breve exposición de la génesis del problema, podemos decir que el origen de la violencia que actualmente experimenta la mujer y el hombre común, reside en la desincronización de la forma del mundo y de su materialización en la ciudad, de la forma del ser humano y su experiencia espiritual.

El homo sapiens, acostumbrado durante milenios a desenvolverse en pequeños grupos vinculados por razones de eficiencia o simbolismo con el territorio que habitaba, experimenta hoy el estrés planetario que causa el desarraigo del territorio, ahora convertido en el plano sobre el que se dibuja una nueva cartografía de fragmentos con relaciones asimétricas.

Desplazarse por las calles cualquier cuidad puede ser una experiencia multidimensional si paseamos a la deriva. Junto a calles llenas de una vida atemporal donde lo local -formado por una suerte de mezcolanza de gentes

THÉMATA. Revista de Filosofía, $\mathrm{N}^{\circ} 48$ julio-diciembre (2013) pp.: 37-48 doi: 10.12795/themata.2013.i48.03 
autóctonas, residentes temporales, multiculturalidad y heterogeneidad- se impone como experiencia de desaceleración, encontramos centros de actividad terciaria y administrativa de escala territorial, y junto a ellos, la sensación de pasar a otra dimensión espacial cuando ingresamos en los centros históricos escenificados para el turismo global. Es como si en un mismo espacio físico transitásemos por distintos cuantums pertenecientes a geografías diferenciadas, sólo comprensibles desde una cartografía discontinua global.

El recorrido por esos espacios temáticos propios de los centros históricos musealizados, nos hablan de una geografía de escala supranacional en la que la lógica del reparto de las cuotas de consumo turístico actúa de manera específica.

Los centros de servicios terciarios y administrativos, nos hablan de geografías en la que las relaciones con los núcleos del hinterland dependiente, con las capitales de provincia y con las sedes administrativas principales, es la que confiere una lógica y un orden regional.

Los centros financieros de las grandes metrópolis participan de unas relaciones a nivel internacional que responden a una lógica geoeconómica absolutamente diferenciada del resto de los fragmentos de la ciudad.

Finalmente, adentrarnos en las calles ocultas del centro histórico, en los barrios de la ciudad consolidada o en las desparramadas periferias urbanas, nos recuerda la dimensión física de nuestro deambular: estamos aquí y ahora. El espacio se comprime respecto a la escala global, y el tiempo se expande.

Así, lo que caracteriza las nuevas cartografías del mundo globalizado son las relaciones equipotenciales entre fragmentos distanciados entre sí tanto como los centros financieros de Londres, Nueva York, Madrid o Tokio, los campos de refugiados de África, los campamentos de afectados por catástrofes naturales, los suburbios autoconstruidos de las grandes aglomeraciones de América del Sur o de Asia, o los barrios del extrarradio de las grandes ciudades europeas, por ejemplo.

Esta geografía discontinua cuenta con múltiples niveles de interpretación, y en una misma ciudad podemos encontrar áreas continuas o discontinuas, e incluso espacios de escala menor dentro de las arquitecturas de los edificios públicos o privados, que hablan de relaciones asimétricas de difícil conexión.

La sofisticación de los códigos espaciales que damos por válidos, generan umbrales diferenciados entre espacios asimétricos que impiden el paso de la población de un lado a otro. Existen límites no necesariamente físicos, que actúan como auténticas barreras sociales. Así, a ninguna inmigrante sin papeles empleada en el servicio doméstico, se le ocurriría entrar a un local de lujo, por más que pudiera pagar la consumición de un café y por mucho que no estuviese prohibida la entrada.

THÉMATA. Revista de Filosofía, $\mathrm{N}^{\circ} 48$ julio-diciembre (2013) pp.: 37-48 doi: 10.12795/themata.2013.i48.03 
Por otra parte, ningún alto ejecutivo de una multinacional se adentraría en un barrio residencial populoso sin su coche, por más que éste no fuese peligroso o por mucho que hubiese locales comerciales con productos que pudiesen ser de su interés.

Existen distintos espacios que alojan a distintos grupos que, por contiguos que sean, muestran distancias insuperables. Por otra parte, el hecho de que para una profesional creativa sea fácil trasladarse en una misma semana a Barcelona, Milán o Nueva York, habla de la existencia de relaciones de equipotencialidad entre fragmentos urbanos distanciados tanto como continentes.

Esta nueva geografía genera conflictos y violencia entre los distintos grupos. Se libra una sorda batalla en el ámbito del territorio que demanda la concepción de un nuevo tipo de justicia: la justicia espacial.

La violencia espacial que hoy sufre la población, sigue dos vectores diferenciados. Por un lado experimenta una limitación en el tránsito de fragmentos pertenecientes a cartografías diferenciadas aunque sean contiguas en el espacio físico.

Por otro, se violenta también a causa del estrés provocado por el obligado desplazamiento de un fragmento a otro de un mismo nivel de cartografía, que sin embargo está distanciado en el espacio físico. La mujer y el hombre común debe transitar por las áreas residenciales, productivas, dotacionales y de ocio, en un continuo desplazamiento espacial a través de las infraestructuras de comunicación física.

Es por ello, que una de las formas más dramáticas y ocultas de discriminación que se producen en la actualidad, sigue estos mismos vectores. Por un lado por la falta de consciencia de la población respecto a ese código espacial que genera umbrales invisibles entre cartografías distintas pero físicamente contiguas. Por otro lado por la limitación del acceso al transporte, pues la necesidad de desplazarse en ese plano equipotencial (de la vivienda al trabajo, a los centros administrativos, dotacionales o de ocio, fruto de la planificación zonificada del urbanismo del siglo XX), pero discontinuo en el espacio hace de ello una condición imprescindible para la vida en la ciudad. La limitación a la libre movilidad genera exclusión social y laboral.

Muchas veces esta limitación no está necesariamente vinculada a la precariedad económica. También aquí las causas de esa discriminación son muy sutiles y en la mayoría de los casos invisibles. Pensemos por ejemplo en las mujeres que no trabajan fuera de casa y que viven en barrios residenciales de clase modesta en los que suele haber un vehículo por unidad familiar. Muchos de estos barrios son de baja densidad, están alejados del centro y no disponen de equipamientos ni dotaciones comerciales. La falta de una masa crítica suficiente hace que las administraciones locales no lleven líneas de transporte público hasta esos barrios, o si lo hacen sea con una importante limitación en los recorridos y frecuencias.

THÉMATA. Revista de Filosofía, $\mathrm{N}^{\circ} 48$ julio-diciembre (2013) pp.: 37-48 doi: 10.12795/themata.2013.i48.03 
Pensemos también en los barrios residenciales de viviendas unifamiliares de alto nivel económico. En ellos tampoco suele haber suficiente frecuencia ni cobertura de transporte público debido a esa misma la baja densidad. Si bien los residentes suelen tener más de dos vehículos por unidad familiar, las personas que trabajan en el servicio doméstico de estos barrios ven limitada su movilidad, dificultando aún más el desempeño de un trabajo que en muchos casos es por horas y exige de un desplazamiento permanente a distintos lugares.

Pero también las personas dependientes como, las personas mayores, las personas enfermas o las personas con movilidad reducida se ven claramente discriminados por un sistema que no ha tenido ni tiene en cuenta la vida de las personas en un nuevo escenario de cartografías fragmentadas en lo espacial y lo social.

Por ello, la guerra de la sociedad tardomoderna no se desarrolla en los tradicionales escenarios de los conflictos bélicos, sino que su marco es el territorio y el campo de batalla el cuerpo de cada individuo.

\section{Hacia una posición.}

Si queremos corregir las asimetrías que generan las nuevas condiciones espaciales del territorio físico y virtual, será necesario construir un nuevo cuerpo teórico que de pie a desarrollar un marco legal que sea consciente y conciencie sobre esta nueva forma de desigualdad social.

Del mismo modo que se ha hecho anteriormente con conceptos como la defensa de la igualdad de género, la protección del patrimonio o del medio ambiente, será necesario explicitar, sensibilizar y politizar la defensa de la justicia espacial para limitar la violencia que las nuevas cartografías globales generan en la experiencia del ser humano en el territorio. Cartografías que se han desarrollado como consecuencia del desarraigo de la mujer y el hombre modernos, del constructo social que era el territorio propio, como resultado de la contradicción que supone la ciudad contemporánea.

Para que este objetivo sea posible, será necesario utilizar las mismas herramientas que a lo largo de la modernidad han ido construyendo sujetos históricos y agentes jurídicos, a la vez que normas que regulen sus relaciones y compensen sus desequilibrios.

Para ello se deberán incorporar de manera transversal en la normativa jurídica, aspectos que tengan por objeto garantizar la aplicación de una justicia espacial que ayude a cada persona a desarrollar la difícil experiencia de la habitabilidad en el territorio, como nueva condición de urbanidad de la ciudad difusa.

THÉMATA. Revista de Filosofía, $\mathrm{N}^{\circ} 48$ julio-diciembre (2013) pp.: 37-48

doi: 10.12795/themata.2013.i48.03 


\section{Bibliografía:}

Zarone, G. (1993). Metafísica de la ciudad. Valencia: Pre-Textos.

Caniggia, G. (1976). Struttura dello spazio antropico. Florencia: Alinea.

Caniggia, G., \& Maffei, G. (1979). Composizione architettonica e tipología edilizia. 1. Lectura dell'edilizia di Base. Venecia: Marsilio.

Se ha tenido acceso a dichas lecturas a través de Luque Valdivia J, "Una teoría arquitectónica de la ciudad. Estudios tipológicos de Gianfranco Caniggia, en revista" RE Revista de Edificación n ${ }^{0} 16$ pp 79-84 año 1993, en $n^{\circ} 17$, pp 75-80

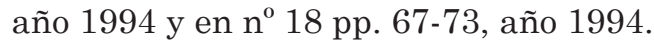

Gómez Zotano, J. (2003). "El papel de los espacios montañosos como traspaís del litoral mediterráneo andaluz: el caso de Sierra Bermeja (Málaga)". Tesis Doctoral . Granada: Dpto. Análisis Geográfico y regional, y Geografía Física. Secchi, B. (1986). Las condiciones han cambiado. Casabella. 